\title{
The Effects of Circuit Training and Circuit Training with Whole Body Vibration on Pulmonary Function in Adolescent
}

\begin{abstract}
The purpose of this study was to parallel circuit training and circuit training with sonic systemic mechanism was to compare the differences in pulmonary function and chest expansion in adult men. This study was performed on 20 subjects. 20 subjects were divided into two groups; Circuit training group $(n=10)$, Circuit training with sonic systemic mechanism $(n=10)$. Both of the group performed the exercise 3 times a week for 5 weeks. The data was analyzed by the Repeated t-test for comparing before, during and after changes of factors in each group and the Independent t-test for comparing the between groups. The result are as follows. Circuit training group was statistically significant difference FVC, FEV1/FVC(p<.05), Circuit training with sonic systemic mechanism group was statistically significant difference PEF, VC in pulmonary function(p<.05). Circuit training group was statistically significant difference FEV1/FVC of between the two group in pulmonary function(p<.05). Circuit training group and circuit training with sonic systemic mechanism group was statistically significant difference in chest expansion $(p<0.05)$ and there was no statistically significant difference of between the two group in chest expansion(p $>$.05).
\end{abstract}

Key words: Pulmonary Function; Circuit Training; Sonic Systemic Mechanism
Hyun ju Juna, Chan Joo Jeonga, Hoe Song Yanga, Ye rim Jeong, Hyuk Jegal ${ }^{a}$, Young Dae Yoo ${ }^{a}$

${ }^{a}$ Cheongam College, Suncheon, Korea

Received : 20 June 2015

Revised : 18 July 2015

Accepted : 02 september 2015

Address for correspondence

Young Dae Yoo, PT, Ph.D

Department of Physical Therapy, Cheongam College, 1641 Noksaek-ro,

Suncheon, Korea

Tel: 82-61-740-7331

E-mail: ptyoo@scjc.ac.kr

\section{INTRODUCTION}

Micro-dust have been increased the incidence rate of heart and pulmonary disease, and mortality also can be numeric increasing(1).

Breathing is the basis for all life support and physical activity, And it affects the recovery in motor function. So that, The movement for improved respiratory function is the most basic and critical interventions(2). Breathing exercise can lead to increase exhalation, the strength of respiratory muscle, coordination, pulmonary function. As a result, It can be improving their activity of daily living(3).

Weakness of respiratory muscle may be needed an intervention which can be increased motor performance, breathing capacity(4). Respiratory muscle can be increased through aerobic exercise to its endurance, strength and pulmonary function(5).

Circuit training is a prescription of exercise which an anaerobic resistance exercise is added with aerobic factor for improving general health status including a cardiopulmonary and a musculoskeletal system on health adult, athletic, cardiac patient(6).

Whole body vibration is a neuromuscular training to improve the morphological and functional development of the physiological factors, muscle fiber by their intrinsic weight and movement to different positions without external resistance on the platform to cause mechanical irritation(7). Whole body vibration exercise brought increasing the motor unit co-activation, accompanied by increased contracted capacity, antagonist stimulation. as a result, exercise performance such as agility, muscle strength, muscle power has increased(10). Recently, Whole body vibration is more convenience and safe, and it has reported that it can affect on musculoskeletal, endocrine, circulatory system as new exercise prescription. and Whole body vibration exercise benefits can be easily without the joint motion, and although exercise time is short, exercise consumption is a lot. 
therefore, it can be effective to prevent an obesity(8).

Inhalation exercise for improving pulmonary function can be helped to improve an endurance and strength of diaphragm and inhalation muscle through loading those muscles.

This basic principles are applied to the skeletal muscle strength training based on the overload, a reversible, specific(9). Therefore, We investigated the effect of caridopulmonary function due to strength of the breathing muscle, and influence according to period through circuit training and circuit training with WBV, compared the pulmonary function between groups.

\section{METHODS}

\section{Subjects}

Subjects were selected 20 male university students who has no musculoskeletal, bronchial diseases and no receive PT within 6 months in Suncheon city. They were randomly divided into two groups; Group 1(circuit training group:10 people), Group 2(circuit training with Whole Body Vibration(WBV) group:10 people). The general characteristic of subjects are follow(Table 1).

Table 1. General characteristic of experimental

\begin{tabular}{ccc} 
subjects & & $(\mathrm{N}=20)$ \\
\hline Division & Group1( $\mathrm{n}=10)$ & Group2 $(\mathrm{n}=10)$ \\
\hline Age $(\mathrm{yr})$ & $21 \pm 1.25$ & $22.7 \pm 3.06$ \\
Height $(\mathrm{cm})$ & $171.8 \pm 3.39$ & $172.6 \pm 4.95$ \\
Weight $(\mathrm{Kg})$ & $64.55 \pm 9.32$ & $69.1 \pm 9.96$
\end{tabular}

$\mathrm{M} \pm \mathrm{SD}:$ Mean \pm Standard deviation

Group1 : circuit training group

Group2 : circuit training with WBV group

\section{Measurement Tools and Measuring Methods}

Subjects were selected 20 people, and they randomly divided circuit training group $(\mathrm{N}=10)$, circuit training with WBV group $(\mathrm{N}=10)$. each groups were conducted warming up exercise(5 minutes), main exercise(20 minutes), resting time(10 minutes) and cool down exercise(5 minutes). Totally, it was conducted 3 times a week, 5 weeks.

Before real training, the subject followed VCR training program, and then they were trained breathing exercise. In order to analyzed difference between groups, we compared to vital capacity and expansion of chest wall before during and after using Spirometry per PC.

\section{Measurement for spirometry}

Spirometry per PC were measured as forced vital capcity(FVC), forced expiratory volume at one sencond(FEV1), FEV1/FVC, peak expiratory flow(PEF), vital capacity(VC). Subjects were measured their body weight, height, and the data was input spirometry and measurement was done having mouthpiece.

\section{Measurement for expansion of chest wall}

Subjects were required exhale as much as they can. At the same time, Inspector measured the tape line which covers between 4th and 5th ribs on chest, and then required inhale as much as possible once again, After that, They hold breathing inspector measured subjects' chest circumference, so that inspector can compare a difference length as inhalation and exhalation. its average is $3 \sim 7.5 \mathrm{~cm}(10)$.

\section{Data Analysis}

The significance test for yield data within group was used to repeated measured ANOVA. If each groups has a difference, Significance was analyzed using Repeated Contrast test. Significance test of inter group was used to Independent T-test. Significance level was set up .05( $\alpha=.05)$.

\section{RESULTS}

\section{The Change of FVC according to Period}

There was significant difference according to period in each groups. as a result of repeated contrast test for interval measurement period in each groups(Table 2), circuit training group had no difference in circuit training group after 3 week $(p\rangle .05)$, but had significant difference after 5 week $(p<.05)$. In circuit training with WBV group had no significant difference after 3 , and 5 week(p>.05).

\section{The Change of FEV1 according to Period}

There was no significant difference measurement period(p>.05), and interaction between measurement period and subjects(p〉.05)(Table 2). 


\section{The Change of FEV1/FVC according to Period}

There was significant difference according to period in each groups. as a result of repeated contrast test for interval measurement period in each groups(Table 2), circuit training group had no difference after 3 weeks(p $\rangle .05)$, but had significant difference after 5 week $(p<.05)$. In circuit training with WBV group had no significant difference after 3 and 5 week $(p>.05)$.

\section{The Changes of PEF according to Period}

There was significant difference according to period in each groups. as a result of repeated contrast test for interval measurement period in each groups(Table 2), circuit training group had no difference after 3 weeks(p $\rangle .05)$, but had significant difference after 5 week(p<.05). In circuit training with WBV group had no significant difference after 3 and 5 week(p〉.05).

\section{The Change of VC according to Period}

There was significant difference according to period in each groups. as a result of repeated contrast test for interval measurement period in each groups(Table 2), Circuit training group had no significant difference after 3 and 5 week(p $\rangle .05)$. In circuit training group with WBV group had no difference after 3 weeks(p>.05), but had significant difference after 5 week(p<.05).

\section{The Change of Expansion of Chest Wall according to Period}

There was significant difference according to period in each groups. as a result of repeated contrast test for interval measurement period in each groups(Table 2), circuit training group had no difference after 3 weeks(p $\rangle .05)$, but had significant difference after 5 week $(\mathrm{p}<.05)$. In circuit training with WBV group had no difference after 3 weeks(p $>05)$, but had significant difference after 5 week(p<.05).

Table 2 Changes of pulmonary function according to period

\begin{tabular}{ccccccc}
\hline \multirow{2}{*}{$\begin{array}{c}\text { Pulmonary } \\
\text { function }\end{array}$} & \multicolumn{2}{c}{ Pre } & \multicolumn{2}{c}{3 weeks } & \multicolumn{2}{c}{5 weeks } \\
\cline { 2 - 7 } & Group1 & Group2 & Group1 & Group2 & Group1 & Group2 \\
\hline FVC & $3.90 \pm .53$ & $4.20 \pm .67$ & $3.98 \pm .45$ & $4.19 \pm .60$ & $4.15^{*} \pm .57$ & $4.33 \pm .63$ \\
FEV1 & $3.43 \pm .56$ & $3.27 \pm .63$ & $3.66 \pm .38$ & $3.30 \pm .68$ & $3.58 \pm .45$ & $3.74 \pm .29$ \\
FEV1 / FVC & $87.64 \pm 8.74$ & $78.66 \pm 15.13$ & $92.01 \pm 5.42$ & $79.29 \pm 16.11$ & $86.76^{*} \pm 8.00$ & $87.46 \pm 9.55$ \\
PEF & $6.98 \pm 3.60$ & $6.93 \pm 4.12$ & $7.47 \pm 1.07$ & $6.06 \pm 2.40$ & $7.30 \pm 1.62$ & $8.06^{*} \pm 2.12$ \\
VC & $3.38 \pm .49$ & $3.59 \pm .85$ & $3.26 \pm .69$ & $3.25^{*} \pm .89$ & $3.35 \pm .76$ & $3.79 \pm .59$ \\
Expansion of chest wall & $6.88 \pm 1.82$ & $6.25 \pm 1.69$ & $6.70 \pm 1.57$ & $6.46 \pm 1.59$ & $9.20^{*} \pm 1.67$ & $9.55^{*} \pm 2.97$ \\
\hline
\end{tabular}

$\mathrm{M} \pm \mathrm{SD}:$ Mean \pm Standard deviation

Significance was tested by repeated-ANOVA(* : p<.05)

Group1 : circuit training group

Group2 : circuit training with WBV group

FVC : Forced Vital Capacity

FEV1: Forced Expiratory Volume at one second

PEF : Peak Expiratory Flow

VC : Vital Capacity

FBTG : Feedback Breathing Training Group

BBTG : Ballon-Blowing Training Group

\section{DISCUSSION}

We investigated to compare a difference at pulmonary function and expansion of chest wall through circuit training and circuit training with WBV on 20 adult male subjects.

This study has shown that there were a significant difference the FVC, FEV1, FEV1/FVC, and expansion of chest wall to comparison of vital capacity according to period in circuit training group.

In previous research, Twenty women was selected, and divided into two groups; one group was conducted circuit 40 minutes, another group was conducted cycling with circuit training considering 
their maximal exercise capacity. In previous research, 30th women were conducted in circuitweight training 4 times a week, 12 weeks. The training time was 30 40 minutes. exercise intensity had been changed during 12 weeks. As a result of stress test using treadmill, there were a significant improvement in their maximal heart rate, maximal breathing rate, forced expiratory volume at one time(11). as well as, the systemic exercise for considering which are appropriate to private's condition, prolonged duration has been expected positive effects in terms of pulmonary function and body composition based on the previous research.

Breathing muscles such as diaphragm, intercostalis and scalenes should be used, especially the diaphragm can be responsible $60 \sim 70 \%$ as inspiration, and as expiration, diaphragm can used by passively relaxation(12). In order to be expansion of chest wall, breathing muscle including diaphragm should be supported. These results can be assigned to mean a significant increase in the expansion of chest wall in this study. In this study, circuit training with WBV group was significance different the expansion of cheat wall and maximal expiratory speed and vital capacity at the compared period.

Vibration program which can be selected 2 specific motion was conducted on twenty adult male using vibrator 5 times a week, 12 weeks. One motion among vibration program was conducted 60 sec, breaking time $30 \mathrm{sec}$, totally 6 times repeated, $20 \mathrm{~Hz}$ of vibration frequency, $26 \mathrm{~Hz} 5 \sim 8$ weeks, $32 \mathrm{~Hz}, 9 \sim 12$ week. the frequency was increased progressively. This study has shown that there are a significantly increased oxygen carrying capacity and utilization by result of exercise(13). although this study was conducted only 5 weeks, there was no negative effects. However, if the program was consisted of more effective including vibration intensity, we could expect more positive effects. Either, we were thought that if considering maximal heart beat, its effects might be different.

In previous research, 14 people male(control group: 7, experimental group: 7) were participated 3times a week, 8weeks. as a result, experimental group had shown that changing of their composition, improving their oxygen saturation due to increase their circulation for conducting prolonged whole body vibration. Therefore, whole body vibration can be an exercise which limits excessive ascension of blood lactate concentration and thereby, it has a strong resistance to fatigue(14). previous study has advocated that the whole body vibration can influence the fatigue rate, so it can be helpful to improve exercise capacity.

The rate of FVC, FEV1, FEV1/FVC are used as indicator to estimate the inprovement of vital capacity, functional levels of breathing muscles, ability to expand chest wall(15). After expansion chest wall exercise, Chronic obstructive pulmonary disease patient has shown their pulmonary function and level of dyspnea has a significant improvement(16). Expansion chest wall exercise is emphasized expiration and depth of inspiration by active movement of trunk and extremity. as a result, it can be increased the movement of between the ribs and release the stiff connective tissues. Expansion chest wall exercise also is one of intervention, which can be released the pectoralis major, intercostalis, quadratus lumborum(17). After the exercise, there was a significantly increased in expansion of chest wall in this study. This aerobic exercise can have an effect not only a neurologic patient but also young adult.

Stroke who has lowered a breathing function can be easy fatigue as they perform aerobic exercise which needs the endurance(18). But increasing of respiratory function showed improvement in walking ability and the results of the independent activities of daily living(19). If rehabilitation program which is integrated with breathing for improving functional activity such as gait in stroke, cardiopulmonary patient, it can be expected positive effects(20,21). Aerobic exercise using treadmill has positive effect on gait speed, endurance and pulmonary function in stroke(22). Like this, improvement of breathing function can be affected in our activity of daily living positively. we expect that significant improvement of circuit training and circuit training with $\mathrm{W} \mathrm{B} \mathrm{V}$ can be influenced not only breathing function but also quality of life.

But the exercise was conducted only 5 weeks. that is, applied duration is short and subjects did not have an opportunity for their maximal capacity on limited base of support. So if considering enough base of support to perform the exercise on clinic, its effect can expect that it is helpful to improve cardiopulmonary function. The previous study has mentioned that stroke who has limited their activity of daily living needs cardiopulmonary capacity for controlling the trunk and gait ability consistently(23).

Consequently, the circuit training program can helpful to improve the quality of life beyond lung 
function in previous and present study. and we hope that comprehensive program including circuit and circuit with WBV may develop.

\section{CONCLUSION}

We compared the influence the pulmonary function followed by measurement period and intergroups pulmonary function of circuit training and circuit training with WBV.

In compared the measuring time, circuit training group had a significant difference the FVC, FEV1/FVC and vital capacity, and circuit training group had a significant difference on the PEF, VC. In compared on between group, the rate of FEV1/FVC had only a significant difference. In compared expansion of chest wall, There were a significant difference in circuit training groups, and Circuit training with WBV had significant difference within group according to period, but no significant difference between group.

In this study, young adult male has shown positive effects. therefore we can mention to develop various program considering aging and body characteristic. As well as if might consider the strength of vibration and body specificity. we also expect that it has more positive effects.

\section{REFERENCES}

1. Kim HJ. Burden of disease due to outdoor air pollution in Korea : based on PM 10. Graduate School of Public Health, Master' s thesis 2014.

2. Oh DH. The Effect of Inspiratory Muscle Training on Pulmonary Function, Deep Abdominal Muscle Thickness and Balance in Patients with Stroke. Graduate School of Sahmyook University, Master' $\mathrm{s}$ thesis 2014.

3. Finnerty JP, Keeping I, Bullough I, Jones J. The Effectiveness of Outpatient Pulmonary Rehabilitation in Chronic Lung DiseaseA Randomized Controlled Trial. Chest 2001; 119(6):1705-1710.

4. Weiner P, Magadle R, Beckerman M, Weiner M, Berar-Yanay N. Comparison of specific expiratory, inspiratory, and combined muscle training programs in COPD. Chest,2003; 124(4):13571364.

5. Carr M, Jones J. Physiological effects of exercise on stroke survivors. Topics in stroke rehabilitation 2003; 9(4):57-64.

6. Kim BK, Park IS, Choi KH. Effect of circuit weight training on body composition physical fitness in the adult with obesity. JKEIA 2014; 5(1):291-294.

7. Ahlborg L, Andersson C, Julin P. Whole-body vibration training compared with resistance training: effect on spasticity, muscle strength and motor performance in adults with cerebral palsy. J Rehabili Med 2006; 38(5):302-308.

8. Lee SA. phenomenological study on participating experience of whole body vibration exercise. Graduate School of Konkuk University, Master' s thesis 2012.

9. Moodie L, Reeve J, Elkins M. Inspiratory muscle training increases inspiratory muscle strength in patients weaning from mechanical ventilation: a systematic review. Journal of physiotherapyr 2011; 57(4):213-221.

10. Magee David J. Orthopedic physical assessment, 6th Ed. Korea, Elsevier Health Sciences 2014.

11. Kim JH. Effect of aerobic dance and circuit weight training on health-related physical fitness, body composition and cardiopulmonary function in obese women. Graduate School of Kyungsung Unoversity, Master' s thesis 2003.

12. Khedr EM, El Shinawy O, Khedr T, Awad EM. (2000). Assessment of corticodiaphragmatic pathway and pulmonary function in acute ischemic stroke patients. European Journal of Neurology 2000; 7(5):509-516.

13. Jeong DJ. The Effect of Vibration Exercise on Oxygen Transport Capacity. Graduate School of Dong-A University, Master' s thesis 2007.

14. Choe MY. The Effects of Whole Body Vibration Exercise on the Oxygen Consumption, Blood Lactate and Body Composition. Graduate School of Dong Shin University, Master' s thesis 2007.

15. Kisner C, Colby LA. Therapeutic exercise: foundations and techniques, 6th Ed. philadelphia, pennsylvania, FA Davis 2012.

16. Leelarungrayub D, Pothongsunun P, Yankai A, Pratanaphon S. (2009). Acute clinical benefits of chest wall-stretching exercise on expired tidal volume, dyspnea and chest expansion in a patient with chronic obstructive pulmonary disease: a single case study. Journal of bodywork and movement therapies 2009; 13(4):338-343.

17. Frownfelter D, Dean,E. Cardiovascular and Pulmonary Physical Therapy: Evidence to Practice, 5th Ed. USA, Elsevier Health Sciences 2014 
18. Lanini B, Bianchi R, Romagnoli I, Coli C, Binazzi B, Gigliotti F, Scano G. Chest wall kinematics in patients with hemiplegia. American journal of respiratory and critical care medicine 2003; 168(1):109113.

19. Katz-Leurer M, Shochina M, Carmeli E, Friedlander Y. The influence of early aerobic training on the functional capacity in patients with cerebrovascular accident at the subacute stage. Archives of physical medicine and rehabilitation 2003; 84(11):1609-1614.

20. Courbon A, Calmels P, Roche F, Ramas J, Rimaud D, Fayolle-Minon I. Relationship between maximal exercise capacity and walking capacity in adult hemiplegic stroke patients. American journal of physical medicine \& rehabilitation 2006; 85(5):436442.
21. Rognmo $\varnothing$, Hetland E, Helgerud J, Hoff J, Slø rdahl SA. (2004). High intensity aerobic interval exercise is superior to moderate intensity exercise for increasing aerobic capacity in patients with coronary artery disease. European Journal of Cardiovascular Prevention \& Rehabilitation 2004; 11(3):216-222.

22. Macko RF, Ivey FM, Forrester LW, Hanley D, Sorkin JD, Katzel LI, Goldberg AP. Treadmill exercise rehabilitation improves ambulatory function and cardiovascular fitness in patients with chronic stroke a randomized, controlled trial. Stroke 2005; 36(10):2206-2211.

23. Duarte E, Marco E, Muniesa JM, Belmonte R, Diaz P, Tejero M, Escalada F. Trunk control test as a functional predictor in stroke patients. J Rehabil Med 2002; 34(6):267-272. 